\title{
cmaJOPEN
}

\section{Physician perspectives on delays in cancer diagnosis in Alberta: a qualitative study}

\author{
Anna Pujadas Botey PhD MSc, Kathy GermAnn PhD MSc, Paula J. Robson PhD, Barbara M. O’Neill MBA, \\ Douglas A. Stewart MD
}

Abstract

Background: Delays in cancer diagnosis have been associated with reduced survival, decreased quality of life after treatment, and suboptimal patient experience. The objective of the study was to explore the perspectives of a group of family physicians and other specialists regarding potentially avoidable delays in diagnosing cancer, and approaches that may help expedite the process.

Methods: We conducted a qualitative study using interviews with physicians practising in primary and outpatient care settings in Alberta between July and September 2019. We recruited family physicians and specialists who were in a position to discuss delays in cancer diagnosis by email via the Cancer Strategic Clinical Network and the Alberta Medical Association. We conducted semistructured interviews over the phone, and analyzed data using thematic analysis.

Results: Eleven family physicians and 22 other specialists (including 7 surgeons or surgical oncologists, 3 pathologists, 3 radiologists, 2 emergency physicians and 2 hematologists) participated in interviews; 22 were male (66.7\%). We identified 4 main themes describing 9 factors contributing to potentially avoidable delays in diagnosis, namely the nature of primary care, initial presentation, investigation, and specialist advice and referral. We also identified 1 theme describing 3 suggestions for improvement, including system integration, standardized care pathways and a centralized advice, triage and referral support service for family physicians.

Interpretation: These findings suggest the need for enhanced support for family physicians, and better integration of primary and specialty care before cancer diagnosis. A multifaceted and coordinated approach to streamlining cancer diagnosis is required, with the goals of enhancing patient outcomes, reducing physician frustration and optimizing efficiency.

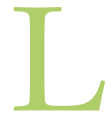

onger time from recognition of a first symptom to diagnosis of cancer is associated with reduced survival, decreased quality of life after treatment and suboptimal patient experience..$^{1,2}$ In Canada, family physicians make important contributions to the care of people with cancer throughout the care continuum., ${ }^{3,4}$ Academic discussions related to this topic have focused on providing clarity about the role of family physicians, and on identifying challenges pertaining to the provision of cancer care in the community. ${ }^{3,5-7}$ However, the emphasis has been mainly on postdiagnostic care, with a particular focus on transitions from specialty cancer care back to the community. ${ }^{6}$ Less attention has been paid to the time before diagnosis. In particular, the processes of handling cancer suspicion and referring to specialists, and how these factors impact timelines to diagnosis remain poorly understood. ${ }^{8,9}$ Little has been published regarding specialist perspectives on delays during the diagnostic period for cancer. ${ }^{3}$

We designed this study to help address these gaps. The objective was to explore the perspectives of a group of family physicians and other specialists in Alberta, Canada, regarding factors contributing to unnecessary delays between first appointment with a family physician and cancer diagnosis (i.e., diagnostic interval), and to solicit their suggestions for expediting or improving the process.

\section{Methods}

\section{Design}

We conducted a qualitative study using interviews that followed an interpretive description approach. ${ }^{10}$ Interpretive description studies explore a clinical phenomenon of interest to capture themes and patterns with subjective perceptions, and generate an interpretive description capable of informing clinical understanding and practice. ${ }^{10,11}$ In this way, interpretive description allowed us to explore the diagnostic interval from the perspective of family physicians and other specialists to inform care during this period.

Competing interests: Anna Pujadas Botey, Kathy GermAnn, Paula Robson and Barbara O'Neill report support from Alberta Health Services. Paula Robson also reports her role as board chair of the Canadian Cancer Research Alliance.

This article has been peer reviewed.

Correspondence to: Anna Pujadas Botey, Anna.PujadasBotey@ahs.ca CMAJ Open 2021 November 30. DOI:10.9778/cmajo.20210013 


\section{Setting and participants}

We used convenience sampling ${ }^{12}$ to recruit family physicians and other specialists involved in the diagnosis of cancer in Alberta, drawing upon existing connections with members of the Core Committee of the Cancer Strategic Clinical Network $\left(9\right.$ physicians) ${ }^{13}$ and the Alberta Medical Association (13000 physicians). We sent identical emails to these 2 groups, inviting both family physicians and other specialists practising in Alberta. We had no specific requests about type of specialists, other than that potential participants be in a position to discuss cancer diagnosis. We shared study information with potential participants and asked them to contact us if they were interested in participating. In addition, we used snowball sampling, whereby we asked participants to recommend physician colleagues who were potentially interested in participating. ${ }^{12} \mathrm{We}$ tracked the profile of respondents as they contacted us and found no need to deny participation to anybody.

\section{Data source and collection}

We conducted in-depth, semistructured interviews. Interview guides for family physicians and other specialists were identical, except for a section pertaining to the process followed by family physicians when patients presented with symptoms (Appendix 1 and Appendix 2, available at www.cmajopen.ca/ content/9/4/E1120/suppl/DC1). Two authors (A.P.B. and K.G.) developed the interview guides based on the literature and findings from a previous study of patient perspectives. ${ }^{14}$ We pilot tested the guide with 4 participants. Participants provided informed consent before interviews.

One author (K.G.), a qualitative researcher with a $\mathrm{PhD}$ in social science and experience in health services research, conducted the interviews. She had no prior relationships or interactions with the individuals approached for interview. She conducted interviews individually by phone, without the presence of nonparticipants. No repeat interviews were conducted. During each interview, the researcher took field notes to maintain contextual details. Interviews occurred in June to September 2019.

\section{Data analysis}

We accepted additional participants until reaching data saturation, meaning that no new themes emerged as we analyzed the interviews that had already been conducted. ${ }^{15} \mathrm{We}$ audiotaped all interviews, transcribed them verbatim and imported them into NVivo Version 11 (QSR International).

We analyzed all data from family physicians and other specialists together. We performed a thematic analysis ${ }^{11}$ using an inductive, data-driven coding process to reflect on how participants made meaning of their experiences. ${ }^{16}$ The analysis entailed a review of each transcript, identification of initial themes, and ongoing development and refinement of themes as data collection and analysis proceeded. One author (K.G.) organized current themes into a set of codes that were applied to text fragments in the transcripts. To ensure consistency and trustworthiness, ${ }^{16} 2$ authors (K.G., A.P.B.) periodically discussed the interpretation and codes until reaching consensus.

\section{Ethics approval}

This study was approved by the Health Research Ethics Board of Alberta, Cancer Committee (HREBA. CC-10-0163).

\section{Results}

We interviewed 33 participants ( $n=22,66.7 \%$ male), including 11 family physicians and 22 other specialists (Table 1 ). Interviews lasted 20-80 (mean 30) minutes.

Our analysis identified 4 themes and 9 subthemes that described factors contributing to potentially avoidable delays in diagnosis, and 1 theme and 3 subthemes that described suggetions for improvement (Appendix 3 and Appendix 4, available at www.cmajopen.ca/content/9/4/E1120/suppl/ DC1). Themes were common to family physicians and other specialists, and diversity was captured in subthemes. Illustrative quotations for themes and subthemes are provided in Table 2 and Table 3.

\section{Nature of primary care}

\section{Limited cancer training}

Although medical students typically learn some basic information about cancer biology, respondents reported that little is taught about cancer diagnosis and treatment in medical school or residency programs for family medicine.

\section{Generalists and information overload}

Family physicians see patients with a diverse range of problems on a daily basis, but typically encounter relatively few patients with cancer throughout their careers. Furthermore, family physicians reported that they find it increasingly difficult to keep up with the continual outpouring of new information about countless diseases and treatments, including cancer.

\section{Initial presentation}

\section{Poor continuity of care}

Many patients do not have a family physician and instead visit walk-in clinics or emergency departments for sporadic care. Without a continuous history, the persistence and serious nature of signs and symptoms related to cancer can easily be missed.

\section{Fee-for-service model}

The fee-for-service model of family physician remuneration in Alberta may unintentionally incentivize some physicians to see many patients each hour, resulting in short appointments that may preclude completion of thorough histories and physical examinations. Specialists lamented that they occasionally see patients who have not been physically examined; both specialists and family physicians discussed the relevance and necessity of physical examinations to identify insidious cancers when patients present with symptoms. 


\section{Investigation}

\section{Difficulties determining appropriate testing}

As acknowledged by family physicians, without clear guidelines other than for the most common types of cancer that have local or provincial programs to help coordinate care (i.e., breast, lung and prostate cancers in Alberta), family physicians are often challenged to know what tests are required to investigate specific signs and symptoms, especially with cancers typified by nonspecific presenting symptoms. In addition, they find it particularly vexing to determine what specific types of biopsy are required, and how to get them completed expeditiously. Specialists mentioned that they can assist in identifying appropriate testing or determining specific testing requirements, but family physicians reported that accessing other specialists is not always easy.

\section{Long wait lists for (sometimes inappropriate) testing}

Family physicians reported difficulty in expediting testing. Inappropriate testing (i.e., unhelpful or erroneous tests) and limited resources may be partially responsible for relatively long wait times for testing, particularly for tests like computed tomography and magnetic resonance imaging. Both family physicians and other specialists agreed that, for many patients, an early referral to a specialist might be warranted since specialists can generally accelerate testing, especially when a cancer diagnosis is suspected.

\section{Specialist advice and referral}

\section{Difficulties determining appropriate specialists}

As recognized by family physicians, identifying the most appropriate specialist is largely dependent upon having a wide network of physician colleagues, which can be problematic for those with limited contacts. An added difficulty is the increasing number of health care specializations, which makes it harder to determine the most appropriate referral.

\section{Difficulties approaching specialists and barriers to referral} Connecting with specialists for advice and for patient referrals is time-consuming and taxing for family physicians. Some specialists make themselves readily available for early advice, especially when cancer is suspected, but others prefer to be contacted only once family physicians have ordered initial tests and have some idea of a potential diagnosis. The practical barriers that family physicians most often mentioned included the low time availability of specialists for consultations, specialists not taking calls, lack of consistent intake approaches, and referral faxes or letters getting lost.

\section{Referral patterns and access to testing}

Physicians work hard to maintain their reputation for providing good and timely care, and they spend part of their career building referral patterns. However, delays are created if

\begin{tabular}{|c|c|}
\hline Characteristic & No. (\%) of participants* \\
\hline \multicolumn{2}{|l|}{ Gender } \\
\hline Female & $11(33.3)$ \\
\hline Male & $22(66.7)$ \\
\hline \multicolumn{2}{|l|}{ Role or specialty } \\
\hline Primary care & $11(33.3)$ \\
\hline Surgery or surgical oncology (breast, gastrointestinal, thoracic) & $7(21.2)$ \\
\hline Pathology & $3(9.1)$ \\
\hline Radiology or diagnostic imaging & $3(9.1)$ \\
\hline Hematology & $2(6.1)$ \\
\hline Emergency medicine & $2(6.1)$ \\
\hline Gynecologic oncology & $1(3.0)$ \\
\hline Medical oncology & $1(3.0)$ \\
\hline Otolaryngology & $1(3.0)$ \\
\hline Public health & $1(3.0)$ \\
\hline Respirology & $1(3.0)$ \\
\hline Years in practice, mean $\pm S D$ & $18 \pm 10$ \\
\hline \multicolumn{2}{|l|}{ Geographical location of practice $†$} \\
\hline Large urban centre & $27(81.8)$ \\
\hline Midsize urban centre & $5(15.2)$ \\
\hline Rural centres & $1(3.0)$ \\
\hline
\end{tabular}


physicians refer patients only to colleagues within their informal networks, without considering other specialists whose wait times may be shorter. Both family physicians and specialists noted that access to testing differs among physicians, potentially contributing to inequities among patients. Although some physicians have managed to get rapid access, others might have to wait long periods.

\section{Suggestions for improvement}

\section{System integration}

Family physicians and other specialists emphasized the need to address system fragmentation by adopting an interdisciplinary, team-based approach that facilitates access to contacts, advice, testing and referrals, thus expediting patient care in a seamless manner.

\section{Care pathways}

Participants referred to clear and seamless standardized pathways for most common cancers as tools that could help manage patient care. Pathways enhance care coordination, set care expectations, and provide cancer-specific recommendations, processes and time frames for patients. In addition, they may include links to resources for clinicians, patients and families (e.g., psychosocial resources, system navigation supports). In this study, physicians described optimal pathways as having embedded centralized and coordinated diagnostic services that are ideally provided at a single location where patients could undergo testing and meet with specialists for a definitive diagnosis.

\section{Centralized advice, triage and referral service}

Participants suggested a single point of entry for family physicians to access supports for diagnosis and referral. Suggestions

Table 2 (part 1 of 2): Perceived factors affecting delays in diagnosis of cancer

\begin{tabular}{|c|c|c|}
\hline Themes & Subthemes & Representative quotations \\
\hline \multirow[t]{2}{*}{$\begin{array}{l}\text { Nature of primary } \\
\text { care }\end{array}$} & Limited cancer training & $\begin{array}{l}\text { "The biggest problem is that most doctors, both specialists and general practitioners, } \\
\text { have no oncology training and the oncology training that they have is directed mostly to } \\
\text { classroom work on the very detailed idiosyncrasies of cancers so the genetics, the parts } \\
\text { of it that people really won't have to use as GPs because they're not specialists. Most } \\
\text { docs have no idea how to diagnose cancer, and they really don't know what to do with it } \\
\text { when they get it. Some of the cancers are getting better. Bowel cancers are getting more } \\
\text { publicity, prostate maybe but by and large, it's really now a dog's breakfast as to what you } \\
\text { know and how you manage it so they essentially turf it to the oncology world [...]. From a } \\
\text { GP point of view, the biggest barrier is an understanding of the disease itself and that's } \\
\text { an education thing." [FP-7] }\end{array}$ \\
\hline & $\begin{array}{l}\text { Generalists and } \\
\text { information overload }\end{array}$ & $\begin{array}{l}\text { "It depends on the family doc, but you have to realize that a lot of family docs may only } \\
\text { see one cancer in their practice, in their life, in their career [...]. I see cancer 24/7, right? } \\
\text { You sort of think it's everywhere, but it's not." [SP-10] } \\
\text { "[Things are getting more complex] and there are more different tests we have to do and } \\
\text { more drugs. You know, when I was a lad, there were four different drugs to treat diabetes. } \\
\text { It's just massive the numbers now and you've got to know all about those." [FP-6] }\end{array}$ \\
\hline \multirow[t]{2}{*}{ Initial presentation } & Poor continuity of care & $\begin{array}{l}\text { "Lack of having a dedicated family doctor is a problem. Certainly, we see big delays in } \\
\text { people that go from walk-in clinic to walk-in clinic with no continuity of care. So, you know, } \\
\text { often people have symptoms and I think if they're seeing the same physician each time, } \\
\text { [that physician] would realize that they're progressing and that there must be something } \\
\text { more significant going on. But, in the walk-in clinics, I don't know if sometimes it's just } \\
\text { another prescription for antibiotics and, 'See ya' So, that's a big problem." [SP-7] }\end{array}$ \\
\hline & Fee-for-service model & $\begin{array}{l}\text { "Patients need a good family doctor, and that's the problem. We have a system that's set } \\
\text { up to make it very difficult to be a good family doctor, because the payment system is } \\
\text { fundamentally set up for seeing six patients an hour. And to actually engage with people } \\
\text { properly, you need to take more time. You need to actually hear what people are } \\
\text { concerned about; you need to tune in to vague stories. It's easy to just do a quick } \\
\text { ten-minute consultation when someone is just coming with a sore throat or even to } \\
\text { diagnose pneumonia. But when somebody comes in and they're looking really sick. } \\
\text { They've got a cough and a fever. You can diagnose and treat that in ten minutes. But } \\
\text { when you're talking about vague, uncertain symptoms, you've got to tease out the } \\
\text { problem and think through issues. That takes time and energy, and the system isn't set } \\
\text { up to allow that. And family doctors who do that are doing it at a cost in terms of } \\
\text { finance." [FP-6] } \\
\text { "There are patients who present with very obvious symptoms, but you have to examine } \\
\text { them. Cancer can be really obvious and sometimes it can be really insidious, and you } \\
\text { have to do a real thorough history [...]. The most important thing, in my opinion is sitting } \\
\text { down and talking to a patient. Your physical exam is only to either confirm or disconfirm } \\
\text { what you've picked up on a history, in my opinion. In your history you can probably find } \\
\text { most things [...]. One of my first patients was having abnormal stools [...], and so I } \\
\text { examined her and I actually felt a mass." [FP-1] }\end{array}$ \\
\hline
\end{tabular}


Table 2 (part 2 of 2): Perceived factors affecting delays in diagnosis of cancer

\begin{tabular}{|c|c|c|}
\hline Themes & Subthemes & Representative quotations \\
\hline \multirow[t]{2}{*}{ Investigation } & $\begin{array}{l}\text { Difficulties determining } \\
\text { appropriate testing }\end{array}$ & $\begin{array}{l}\text { "I see frustrated family practitioners who, while they're trying to sort out 'Where do I send this } \\
\text { patient?', or try to get an answer, and in the meantime, they order a bunch of tests that are } \\
\text { not helpful or are even unnecessary. So, we waste people's time. We waste resources within } \\
\text { the healthcare system doing things that aren't helpful in coming to a diagnosis." [SP-5] } \\
\text { "For us [family physicians], we know there is a mass; we've got some idea of what it is from } \\
\text { the imaging. Really, I think it's up to the specialist to decide what it is they need. So, in the } \\
\text { end, I had to call the on-call, then I had to call a surgeon on call to get him in. Then, a big } \\
\text { hoo-haw and ultimately the surgeon said, 'No', and the patient actually came in with an } \\
\text { obstruction and [we] sent him to the emerg [...]. At the end of the day, I'm playing ping-pong } \\
\text { between the radiologist and the surgeon. Who wants to do it? I don't know, I think the ball's } \\
\text { in our court a little bit too long here." [FP-11] } \\
\text { "It's confusing because we're not experts in particularly uncommon cancers and } \\
\text { sometimes it's just really hard to know what the next step is." [FP-2] }\end{array}$ \\
\hline & $\begin{array}{l}\text { Long waitlists for } \\
\text { (sometimes } \\
\text { inappropriate) testing }\end{array}$ & $\begin{array}{l}\text { "Most of the time patients present with a lymph node in the neck or armpit or groin, and they } \\
\text { present to a walk-in clinic or GP as the first kind of contact. And then generally what happens } \\
\text { is the GP orders an imaging test, usually an ultrasound, to confirm that there's actual lymph } \\
\text { nodes, which to me is kind of silly because if you can feel it, then it's abnormal but that's what } \\
\text { they do. And they do it to characterize it, and then often the radiology report would say, } \\
\text { 'Please do a CT scan', and so that's fed back to the physician who then orders a CT scan, but } \\
\text { that's not the test we want for the patient. The patient needs a diagnostic biopsy, so the CT } \\
\text { scan is actually not the most appropriate next step, and that often delays things." [SP-4] } \\
\text { "There is not enough budget or new investment into AHS DI to keep up with demand for } \\
\text { CT and MRI to keep waitlists where they are. Waitlists are going up." [SP-20] } \\
\text { "Often, if a family physician has a possible mass that could be a sarcoma, they get an } \\
\text { ultrasound. The ultrasound people say, 'Needs an MRI.' They order an MRI. The MRI is } \\
\text { twelve to eighteen months. Hopefully that's not good enough and someone like me gets a } \\
\text { call or a fax [from the family doctor] and then l'm able to triage that, maybe see them in } \\
\text { my clinic a bit quicker. And then, if my name is on an MRI requisition, I can usually get it } \\
\text { within weeks. I've seen it many times." [SP-17] }\end{array}$ \\
\hline
\end{tabular}

Specialist advice Difficulties determining and referral

appropriate specialists
"Most of it is trying to figure out who do you know and how do you get your patient to that [specialist][...] That's not a good way because what it does is it scares the crap out of new physicians. If you are new to the city or you're a new grad or maybe you're even new in the country, that is so daunting [...]. A lot of doctors, especially those who are out of province, out of country, don't know what to do because they don't have the connections and they didn't do their residency here." [FP-1]

"It would be nice if [family physicians] had some better way of accessing specialists because I know they get very frustrated...especially people who trained elsewhere, that's very challenging.... It's very hard to come to a new system and learn who the people are to talk to or whatever." [SP-2]

Difficulties approaching “There isn't a way for a family doctor to reach out. It's kind of discouraged. My experience in specialists and barriers training as a family doctor is nobody likes to get that phone call. Their day is already packed to referrals 9 to 5 and there's no time to schedule an unscheduled phone call from family medicine asking for advice. So, if you're going to bother a specialist, you've got to have a really good reason. And that puts the family doc in a tough situation, where you're looking for more information but you're scared that if you ask that it might be inappropriate." [FP-3] "[Making referrals] is one of the most confusing, non-cohesive parts of the province because the College [Alberta College of Family Physicians] is clear about what they want, but every specialist doctor kind of takes a different direction about how they do [referrals]." [FP-2] "Urology is one of those where you gotta pick up the phone and derail your entire clinic for an hour and a half to make that happen. You do that for the health of your patient, but it is incredibly disruptive." [FP-1]

Referral patterns and access to testing
"The biggest compliment you can give another provider is to refer them your patient. Physicians work hard to maintain their reputation and provide good care, and see people quickly and they spend their career building referral patterns." [SP-21]

"The difficulty is there are patients who are getting lost in the system, and getting lost in the cold, because they just don't happen to be with the physician who's got the rapid access. So, l'd like us to see a system where every single patient gets treated the same way, has the same opportunity access rapid care, as opposed to just being randomly assigned to somebody who might or might not be able to get you in quickly." [SP-3]

Note: AHS = Alberta Health Services, CT = computed tomography, DI = diagnostic imaging, FP = family physician, GP = general practitioner, MRI = magnetic resonance imaging, $\mathrm{SP}=$ specialist physician. 
Table 3: Suggestions for accelerated diagnosis of cancer

Suggestions Representative quotations

System integration

"My main thing is figuring out a way for family docs to get reconnected to the system. What I see happening is [that] medicine is obviously evolving and we're realizing team-based care is really important. And, what I see is Alberta Health Services and the specialist services really working on that, and getting on top of that, and working in inter-disciplinary teams and that kind of thing. [...] And then, family medicine is just kind of on its own. We built this system where we're like, 'Okay, family docs are out in the community, you're on your own'. [...] Family medicine is an afterthought." [FP-3]

"Specialists get more and more sub-specialized which is a problem because it leads to fragmentation. [...] We see that - gastroenterologists who only do hepatology with our liver specialists. They don't do inflammatory bowel disease or colonoscopy or gastroscopy." [SP-5]

Care pathways "It would be helpful to have pathways because then, if a family doctor said, 'Look, I have a pathway in front of me here, this is what they're asking me to do. I need this within a certain period of time'. And if we've set expectations in our discussions with surgeons, diagnostic imaging, family docs, then hopefully we start to get rid of those unnecessary tests that are being done. Because that's what's contributing to the wait times, and getting the right tests at the right time for the right patients would actually improve access." [SP-5] "For [family physicians], if it's an abnormality on a mammogram, it's clear where I go. If it's something on a chest $x$-ray, it's clear which way to go. But for the patients where there isn't a program, they really struggle and they're calling surgeons, 'Can you see the patient to do a biopsy?', calling the oncologist on call, 'What do I do? They've clearly got cancer'. And so, they're scrambling around calling several different people in the course of a busy day trying to facilitate something that to me [as a specialist], we need a single point of contact so that we can assist with the triage and the appropriate direction of patients for whatever service is required to get them to a diagnosis." [SP-5] "I think getting the breast health-type clinics for every major type of cancer, and for the "weird and wonderful" that we just don't know, like 'I just feel uneasy, I think something is wrong', the weird stuff [...]. I think that would be a great use of resources. It's confusing because we're not experts in particularly uncommon cancers, and sometimes it's just really hard to know what the next step is..." [FP-2]

"A potentially dual purpose [...] would be some sort of contact or resource [...]. For the breast health programs in Edmonton and Calgary, they have that. The patient is contacted by the program or vice versa, and there's a nurse navigator or someone else that the patient can talk to. Because that is a potential issue that we face in that interval between the suspicious imaging diagnosis made and the patient being told the results of the biopsy. Who is supporting that, who is supporting the patient in that interval?" [SP-20]

Centralized advice, triage and referral service

"A phone consultation system where you've got somebody, just not quite sure the next step to take, and you phone up somebody and get an immediate consult that says, 'Okay, given that, this is what you should do, go in this direction, do those tests'. So, those are very helpful because that helps us get far enough along that we know there is something there or maybe there isn't something there." [FP-6]

"Having access to speak to the appropriate person, and a lot of times maybe that's not even an oncologist yet. Maybe that's a nurse that specializes in cancer care [...]. So I think there's this whole notion of having a number you can call." [FP-2]

"What we really need is a central triage place where we say, 'Here's the chest mass. Here's what it looks like. Here's what it is.' And then, it would be decided who is going to do what and where, what's that going to look like." [SP-19] "If there was a central cancer booking office, for example, referral's gone in, it's been triaged by the appropriate specialist and the ball is in the system. And if there's something like, 'Oh, the specialist thinks that we should have done something more', then they can call us and inform us. We're happy to take that. But I just feel like until you get a proven tissue diagnosis to the "enth" degree, they don't even want to know. Then by that point, it's a little bit delayed." [FP-11]

Note: FP = family physician, SP = specialist physician.

for what this service would offer included phone or online advice about what tests to order, how to get a biopsy, what specialist to refer to and connecting to the right specialist for guidance; organizing necessary tests; and triaging and referring patients to the most appropriate and available specialist. This service was imagined to be particularly helpful for supporting the care of patients with vague presentations or less common cancers.

\section{Interpretation}

This qualitative study contributes to the literature by focusing on perceived impediments to the expeditious cancer diagnosis. Findings showed that, although family physicians play a critical role in early cancer diagnosis, they may face substantial challenges in effectively unravelling nonspecific symptoms, identifying appropriate testing needs, and accessing diagnostic and specialized resources. Findings also showed that family physicians and other specialists often feel they are working in separate silos, yet it is the specialists who hold the knowledge of how best to expedite cancer diagnosis. Results may inform improvements in health system integration and the development of interventions to streamline the diagnostic process.

Our findings are aligned with the handful of previous studies that have explored potentially avoidable delays occurring in the diagnostic interval in Canada, ${ }^{3,17}$ including poor care continuity, and inconsistent communication and collaboration 
between family physicians and other specialists., ${ }^{3,6}$ This study incorporates the perspectives of specialists, particularly that they appreciate the important and challenging role of family physicians in diagnosing cancer and are willing to provide advice and expedite diagnosis if cancer is suspected. These results are relevant in the context of bridging the "two solitudes" of primary and specialist care. ${ }^{4}$

Participant suggestions, namely the implementation of care pathways with further support for family physicians, are important, given the strong promotion of pathways in the Canadian context to guide care of patients with different cancer types. ${ }^{17}$ A successful example is the Alberta Breast Cancer Diagnostic Assessment Pathway, which addresses variation and wait time between discovery of a highly suspicious finding on imaging and referral to a breast program..$^{18}$ Our study supports the importance of such pathways, as recognized by other stakeholders, including patients, ${ }^{14}$ and validates their perceived value among family physicians and other specialists.

Our results suggest the need to explore the development of novel pathways centred on serious, nonspecific symptoms, as done in other countries. ${ }^{19-21}$ This idea is garnering interest around the world, given that almost half of patients with cancer present with vague symptoms. ${ }^{22}$ Some jurisdictions, including the United Kingdom, Denmark and Manitoba, provide rapid referral pathways that facilitate quick access to testing for patients with specific symptoms and cancer types. ${ }^{9,21,23}$ In addition, our findings suggest it might be unrealistic to expect that family physicians know every existing pathway and have them all readily available when required, which may show the need for pathway maps, as used in Ontario (https:// www.cancercareontario.ca/en/pathway-maps) and for further study of how family physicians think about using pathways.

Our findings highlight the need to improve system integration by adopting team-based approaches and by enhancing access to specialty information and appropriate testing. Participants reported a desire for the development and implementation of a centralized service where primary and specialist physicians converge in their roles. This would help address the issue of promptly getting patients to the right provider, even if family physicians do not have a strong informal network of physician colleagues to draw upon. Initiatives such as specialty teleconsultation system $\mathrm{s}^{24}$ and diagnostic assessment programs ${ }^{25}$ should be considered.

Action to better support the important role of primary care in the diagnostic interval is particularly relevant in the context of the growing number of cancer cases, ${ }^{26-28}$ and the increased demands put on primary care for more involvement throughout the cancer care continuum. ${ }^{29}$ Future studies should further explore and rigorously assess current and innovative approaches to improve integration between primary and specialist care. Consideration of how different contextual factors (e.g., limited training, fee-for-service model) could be addressed to enhance effectiveness are warranted. Approaches are needed to support the involvement of all key stakeholders in the codesign of pathways, centralized referral and support systems with the goal of optimizing the care of patients with a potential cancer diagnosis.

\section{Limitations}

Given resource constraints, we opted to interview additional physicians rather than to seek participant feedback on their transcripts or summary reports. This allowed us to achieve data saturation, lending greater credibility to findings and richer understanding of physician experiences. An additional limitation is that most participants are men, and gender dynamics might have shaped the interview process and data provided by these participants. ${ }^{30} \mathrm{We}$ did not capture information on whether physicians work in an academic or nonacademic setting. Affiliation with an academic institution may be associated with differences in referral experiences. Lastly, only a few physicians residing outside large urban centres participated in the study, and most of them were from communities near major urban centres. As such, the findings may not reflect the experiences of physicians in rural and remote communities of Alberta.

\section{Conclusion}

In this study, we confirmed that family physicians have an important contribution to make in the timely diagnosis of patients with cancer, but found that expeditious diagnosis is often a complex and time-consuming endeavour. Enhancing support and integration among primary care and specialist physicians, coupled with the development and implementation of an efficient diagnosis coordination system, may lead to improved outcomes and experiences.

\section{References}

1. Brousselle A, Breton M, Benhadj L, et al. Explaining time elapsed prior to cancer diagnosis: patients' perspectives. BMC Health Serv Res 2017;17:448.

2. Neal RD, Tharmanathan P, France B, et al. Is increased time to diagnosis and treatment in symptomatic cancer associated with poorer outcomes? Systematic review. Br 7 Cancer 2015;112:S92-107.

3. Easley J, Miedema B, O'Brien MA, et al. The role of family physicians in cancer care: Perspectives of primary and specialty care providers. Curr Oncol 2017;24:75-80.

4. Grunfeld E. The two solitudes of primary care and cancer specialist care: Is there a bridge? Curr Oncol 2017;24:69-70.

5. Tomasone JR, Vukmirovic M, Brouwers MC, et al. Challenges and insights in implementing coordinated care between oncology and primary care providers: a Canadian perspective. Curr Oncol 2017;24:120-3.

6. Brouwers MC, Vukmirovic M, Tomasone JR, et al. Documenting coordination of cancer care between primary care providers and oncology specialists in Canada. Can Fam Physician 2016;62:e616-25.

7. Kendell C, Decker KM, Groome PA, et al. Use of physician services during the survivorship phase: a multi-province study of women diagnosed with breast cancer. Curr Oncol 2017;24:81-9.

8. Rose PW, Rubin G, Perera-Salazar R, et al. Explaining variation in cancer survival between 11 jurisdictions in the International Cancer Benchmarking Partnership: a primary care vignette survey. BMF Open 2015;5: $\mathrm{e} 007212$.

9. Jensen H, Tørring ML, Olesen F, et al. Cancer suspicion in general practice, urgent referral and time to diagnosis: a population-based GP survey and registry study. BMC Cancer 2014;14:636.

10. Thorne S. Interpretive description. Walnut Creek (CA): Left Coast Press; 2008.

11. Thorne S. Interpretive description - qualitative research applied practice. New York, NY: Rootledge; 2016.

12. Patton MQ. Qualitative research \& evaluation methods. Integrating theory and practice. Fourth ed. Saint Paul (MN): SAGE Publications; 2015.

13. Bond TR, Estey A, Elwi A; Cancer Strategic Clinical Network. Cancer Strategic Clinical Network: improving cancer care in Alberta. CMAf 2019;191:S13-4.

14. Pujadas Botey A, Robson PJ, Hardwicke-Brown AM, et al. From symptom to cancer diagnosis: perspectives of patients and family members in Alberta, Canada. PLoS One 2020;15:e239374.

15. Saunders B, Sim J, Kingstone T, et al. Saturation in qualitative research: exploring its conceptualization and operationalization. Qual Quant 2018; 52:1893-1907. 
16. Miles MB, Huberman MA, Saldana J. Qualitative data analysis: a methods sourcebook. 3rd ed. Thousand Oaks (CA): SAGE Publications; 2014.

17. Leading practices to create a seamless patient experience for the pre-diagnosis phase of care: an environmental scan. Toronto: Canadian Partnership against Cancer; 2018.

18. Laws A, Crocker A, Dort J, et al. Improving wait times and patient experience through implementation of a provincial expedited diagnostic pathway for BI-RADS 5 breast lesions. Ann Surg Oncol 2019;26:3361-7.

19. Lewis R. Improving diagnostic pathways for patients with vague symptoms. Accelerate, Coordinate, Evaluate (ACE) Programme. ACE Vague Symptoms Cluster. London (U.K.): NHS England, Cancer Research UK and Macmillan Cancer Support; 2017

20. Nicholson BD, Oke J, Friedemann Smith C, et al. The Suspected CANcer (SCAN) pathway: Protocol for evaluating a new standard of care for patients with non-specific symptoms of cancer. BMF Open 2018;8:e18168.

21. Vedsted P, Olesen F. A differentiated approach to referrals from general practice to support early cancer diagnosis - the Danish three-legged strategy. Br 7 Cancer 2015;112:S65-9.

22. Walter FM, Rubin G, Bankhead C, et al. Symptoms and other factors associated with time to diagnosis and stage of lung cancer: a prospective cohort study. Br 7 Cancer 2015;112:S6-13.

23. CancerCare Manitoba. Manitoba cancer plan 2016-2021. Winnipeg: CancerCare Manitoba; 2016. Available: https://www.iccp-portal.org/plans/ manitoba-cancer-plan-2016-2021 (accessed 2021 Nov. 15).

24. Arain M, Rostami M, Zaami M, et al. Specialist LINK and primary care network clinical pathways - a new approach to patient referral: a cross-sectional survey of awareness, utilization and usability among family physicians in Calgary. BMC Fam Pract 2020;21:86.

25. Brouwers M, Oliver TK, Crawford J, et al. Cancer diagnostic assessment programs: standards for the organization of care in Ontario. Curr Oncol 2009;16:29-41.

26. Poirier AE, Ruan Y, Walter SD, et al. The future burden of cancer in Canada: long-term cancer incidence projections 2013-2042. Cancer Epidemiol 2019;59:199-207.

27. Canadian Cancer Statistics Advisory Committee. Canadian cancer statistics 2019. Toronto: Canadian Cancer Society; 2019.

28. The Canadian strategy for cancer control: 2019-2029. Doing together what cannot be done alone. Toronto: Canadian Partnership against Cancer; 2019.

29. Rubin G, Berendsen A, Crawford SM, et al. The expanding role of primary care in cancer control. Lancet Oncol 2015;16:1231-72.

30. Outpatient Cancer Drug Benefit Program. Edmonton: Alberta Health Services; 2021.
Affiliations: Cancer Strategic Clinical Network (Pujadas Botey, Stewart), Alberta Health Services, Calgary, Alta.; School of Public Health (Pujadas Botey), University of Alberta, Edmonton, Alta.; Independent health services researcher (GermAnn), Lacombe County, Alta.; Cancer Strategic Clinical Network (Robon, O'Neill), Alberta Health Services, Edmonton, Alta.; Cancer Care Alberta (Robson), Alberta Health Services, Edmonton, Alta.; Departments of Oncology and Medicine (Stewart), University of Calgary, Calgary, Alta.

Contributors: Anna Pujadas Botey and Paula Robson conceptualized the study. Anna Pujadas Botey and Kathy GermAnn designed the study, with support from Paula Robson. Anna Pujadas Botey administered the project, and Kathy GermAnn collected and analyzed data. Kathy GermAnn, Paula Robson, Barbara O'Neill and Douglas Stewart made substantial contributions to the interpretation of data. Anna Pujadas Botey prepared the first draft of the manuscript. All authors revised it critically for important intellectual content, gave final approval of the version to be published and agreed to be accountable for all aspects of the work.

Funding: The authors did not receive specific funding for this work.

Content licence: This is an Open Access article distributed in accordance with the terms of the Creative Commons Attribution (CC BY-NC-ND 4.0) licence, which permits use, distribution and reproduction in any medium, provided that the original publication is properly cited, the use is noncommercial (i.e., research or educational use), and no modifications or adaptations are made. See: https://creativecommons.org/licenses/by-nc-nd/4.0/

Data sharing: All relevant data are within the manuscript. Further data excerpts are available on request from the corresponding author.

Acknowledgements: The authors acknowledge service sections of the Alberta Medical Association for their assistance in recruiting participants, and study participants for their insights and the Core Committee of the Cancer Strategic Clinical Network for their support.

Supplemental information: For reviewer comments and the original submission of this manuscript, please see www.cmajopen.ca/content/9/4/ E1120/suppl/DC1 